\title{
The development of beliefs about falling objects
}

\author{
MARY KISTER KAISER \\ University of Michigan, Ann Arbor, Michigan \\ DENNIS R. PROFFITT \\ University of Virginia, Charlottesville, Virginia \\ and \\ MICHAEL McCLOSKEY \\ Johns Hopkins University, Baltimore, Maryland
}

\begin{abstract}
Previous studies have shown that many adults have striking misconceptions about the motions of objects in seemingly simple situations. The present two studies explored the development of knowledge about motion by examining children's predictions about the movement of an object in two types of situations. In one type of situation, children predicted where a ball would land if it rolled off the edge of a table and fell to the floor. In the other type of situation, children judged where the same ball would land if it were dropped from a moving model train and fell the same distance to the floor. Younger children (preschool and kindergarten) generally thought that the ball would fall straight down in both situations. At older ages, children were more aware that the ball rolling from the table would continue to move forward while falling. For the ball dropped from the train, however, the older children were no more aware of the ball's forward motion than were younger children. The results are interpreted in terms of general cognitive capabilities and perceptual experiences that contribute to the development of knowledge about the world.
\end{abstract}

Recent studies indicate that many people hold striking misconceptions about the natural motions of objects in seemingly simple situations (e.g., Champagne, Klopfer, \& Anderson, 1980; Clement, 1982; McCloskey, 1983a; Viennot, 1979). Figure 1 depicts two situations in which such erroneous beliefs are expressed. On the left is shown a curved tube lying flat on a horizontal surface. When asked to predict the trajectory that a ball would take after being propelled through the tube, many adults report a curved path similar to that drawn with the dotted line (McCloskey, Caramazza, \& Green, 1980). To the right, a plane is shown carrying a capsule. When asked to predict the path that the capsule would take after it had been dropped, many adults report the straight down trajectory illustrated by the dotted line. In this situation, some people even predict that the capsule would fall backward along the path shown by the dashed line (McCloskey, Washburn, \& Felch, 1983). Where do such erroneous beliefs come from, and what role does perception play in their development?

This research was supported by NIMH Training Grant T32-MHI6892 to the first author, and NICHD Grant HD-16195 to the second author. We would like to thank David Larabell, principal of West Willow Elementary School, Steven Sternberg, director of the University of Michigan Children's Center, and their staffs for their hospitality and cooperation. Requests for reprints should be sent to Mary Kaiser, who is now at NASA Ames Research Center, MS 239-3, Moffett Field, CA 94035 .
In investigating these questions, we have found that some beliefs reflect biases inherent to perceptual processing, whereas others do not. In particular, the misconceptions seen in the C-shaped-tube problem do not seem to stem from perceptual influences, whereas the erroneous predictions involving falling objects reflect a bias introduced in the perceptual processing of the ongoing event. Our supposition, investigated in the following studies, is that beliefs that reflect perceptual influences follow developmental courses that are different from those that do not.

\section{The C-Shaped-Tube Problem}

When asked to predict the path that a rolling ball will take upon exiting a $\mathrm{C}$-shaped tube, many adults report that the ball will continue to move along a curved path. When shown contrived video sequences of balls rolling through the $\mathrm{C}$-shaped tube and then following a variety of straight and curved paths upon exit, almost all of these adults state that a ball following the correct straight path looks more natural than balls following curved paths (Kaiser, Proffitt, \& Anderson, 1985). Thus, when viewing the ongoing event, people perceive as natural the correct motion path and not the erroneous trajectories that they predict in representational contexts.

That the misconceptions observed in the C-shaped-tube problem are not primarily due to perceptual biases is also supported by the U-shaped developmental course that adherence to this erroneous belief follows. When asked to 

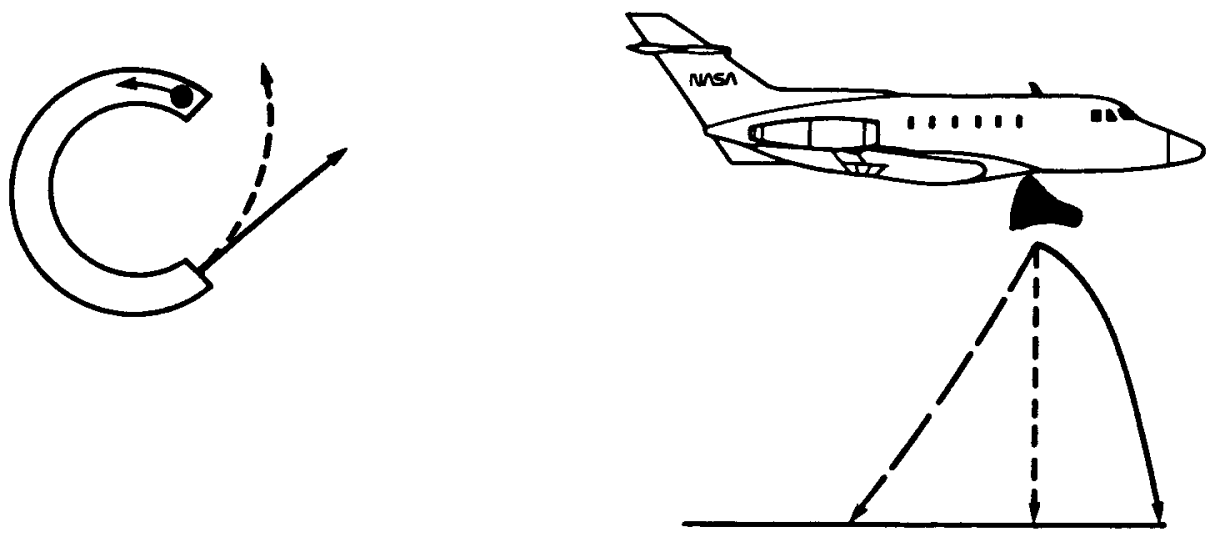

C-SHAPED TUBE PROBLEM

FALLING OBJECT PROBLEM

Figure 1. Motion problems with correct solutions (solid lines) and common incorrect responses (dotted and dashed lines).

predict the path of a ball exiting a C-shaped tube, most children of preschool and kindergarten age predict a straight path, and in this respect perform as well as college students. Correct performance falls off with age until the third and fourth grade, an age at which a large majority of children predict curvilinear motion paths. From fifth grade on, performance improves with age (Kaiser, McCloskey, \& Proffitt, in press).

\section{The Falling Object Problem}

Previous studies on this problem have revealed an interesting mixture of accurate knowledge and misconceptions. Most adults know that an object that moves along an elevated surface and then goes over the edge (e.g., a ball that rolls off a table, a car that runs over the edge of a cliff) continues to move forward as it falls. However, many adults fail to realize that an object that is carried by a moving body and then dropped (e.g., a ball dropped by a walking person, a bomb dropped from an airplane) continues to move forward while falling (McCloskey, 1983a, 1983b; McCloskey et al., 1983). Many adults erroneously predict that the released object will fall straight down. This finding is surprising since, in terms of the relevant physics, the situation involving a carried object and that involving a rolling object are identical. In both cases, the forward and downward motions combine to produce a trajectory that is nearly parabolic. Thus, the path of a carried object that is dropped will be identical to that of an object that rolls off an elevated surface, as long as the two objects are initially moving at the same speed and fall from the same height.

Why, then, do many adults know that objects rolled off elevated surfaces continue forward while falling, but at the same time believe that carried objects fall straight down when dropped? McCloskey et al. (1983) suggested that this misconception was due to a visual illusion in which objects that are dropped are often perceived as falling straight down. McCloskey et al. argued as follows: When people observe carried objects that are dropped (for example, a book dropped by a walking person), the moving carrier (e.g., the walking person) often acts as a frame of reference against which the falling object is viewed. Studies of motion perception (e.g., Hochberg \& Fallon, 1976; Johansson, 1950; Wallach, 1959, 1976) have shown that when an object is viewed against a moving frame of reference, the object's motion relative to the reference frame may be misperceived as its absolute motion (i.e., motion relative to the stationary environment). Ignoring effects of air resistance, an object dropped from a moving carrier falls straight down relative to the carrier, and so may be perceived as falling in a straight vertical path. Experiments show that an illusion of this sort does in fact occur when people observe carried objects that are dropped (McCloskey et al., 1983).

Given that adults hold erroneous beliefs concerning falling objects due to misleading perceptual experiences, one could hypothesize several patterns of development for children's conceptions in this domain. The hypothesis we advance is that young children initially formulate an omnibus belief about falling: "All unsupported objects fall straight down." In most situations that confront a young child, this naive theory would work pretty well, as it captures the most salient aspect of the falling event. Perceptual experience would provide grounds for modifying this belief in the case of rolling objects that fall. However, due to the effects of the moving reference frame illusion discussed above, perceptual experience would be in accord with the child's naive theory in the case of carried objects. Formal training, of course, should force a modification of the omnibus belief in both cases. Empirical support for this hypothesis would include the finding that young children hold to an "all objects fall straight down" belief for both rolling and carried objects. Over the course of development, this belief about falling would change more rapidly for rolling, as opposed to carried, objects.

A reasonable alternative to this hypothesis asserts that children initially formulate an accurate belief about the motion of falling objects. Shepard $(1981,1984)$ has pro- 
posed that, due to the selective pressures of biological evolution, significant dynamic constraints in the physical world are inherent in human motion processing mechanisms. This proposal leads to the hypothesis that accurate naive beliefs might be present early in development, as is observed in C-shaped-tube problems. Misconceptions about the trajectories of carried objects would arise from repeated perceptual experience with the moving reference frame illusion. Empirical support for this hypothesis would include the finding that young children initially hold accurate beliefs about all falling objects and that misconceptions about carried objects develop with age, at least up to the time of formal training.

With respect to either hypothesis, we propose that beliefs that reflect perceptual influences follow different developmental courses from those that do not. Thus, both hypotheses suggest that the developmental course for beliefs about the motions of falling objects will diverge for carried as opposed to rolling objects. With respect to the first hypothesis, perceptual experience should motivate a reformulation of the omnibus "things fall straight down" belief only for the case of rolling objects. With respect to the second hypothesis, perceptual experience should influence the initial accurate belief only in the case of carried objects. Thus, whether early conceptions are accurate or not, beliefs about rolling and carried objects should diverge over development due to the perceptual bias introduced by the moving reference frame illusion.

\section{EXPERIMENT 1}

In this experiment, children and adults were asked to predict the trajectory of a ball rolled off the edge of a table and that of a ball dropped from a moving model train.

\section{Method}

Subjects. The subjects were 100 middle-class children, 4.5 to 12 years of age, and 20 college students. The children were placed into four age groups according to the last grade of school completed. There were 23 preschool and kindergarten children (P\&K; 14 boys, 9 girls), 19 first and second graders ( $1 \& 2 ; 11$ boys, 8 girls), 30 third and fourth graders ( $3 \& 4 ; 12$ boys, 18 girls), and 28 fifth and sixth graders ( $5 \& 6 ; 14$ boys, 14 girls). Nine additional children (five from the P\&K group, four from the $1 \& 2$ group) failed to understand the task and were excluded from the study. The college sample (COL) consisted of 10 men and 10 women, 16 of whom had taken high school or college physics.

Apparatus. An HO-scale model train traveled around an oval track mounted on a table $1 \mathrm{~m}$ above the ground, as shown in Figure 2. A steel ball, $1 \mathrm{~cm}$ in diameter, rode on a flatcar behind the engine. The flatcar was constructed so that the ball could automatically be dropped from the car when it reached a particular point on the track. A section of the table adjacent to the track in this area had been cut out so that the ball, when released, would fall to the floor.

Also mounted on the table, parallel to the train track, was a clear plastic tube that ran to the edge of the cut-out area of the table (see Figure 2). One end of the tube was elevated so that when the $1-\mathrm{cm}$ steel ball was placed in this end, it would roll to the other end of the tube and fall to the floor. The speed of the ball when it emerged from the tube was the same as the speed of the train. A scale-model building hid the elevated end of the tube from the subject's view.

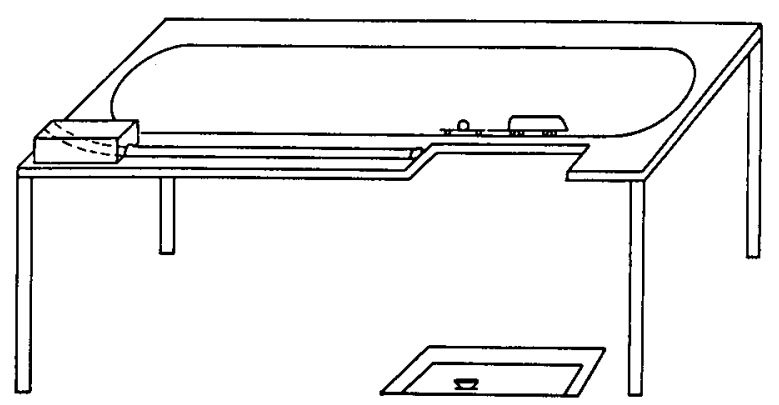

Figure 2. Schematic of experimental layout employed in Experiment 1.

A $28 \times 38 \mathrm{~cm}$ piece of paper was placed on the floor beneath the cut-out area of the table. An outline of the cutout was drawn on the paper to help the subject align points on the table with points on the paper.

Procedure. The subjects were asked to predict the behavior of the ball under three conditions. In the Stationary condition, the subjects were to predict where on the ground the ball would land if it were dropped by a stationary figure standing at the left edge of the cutout. In the Rolled condition, the subjects were to observe the ball moving through the plastic tube and predict where it would land if it rolled out of the tube and fell to the floor. The end of the tube was blocked so that the ball did not actually emerge and fall. Finally, in the Carried condition, the subjects were to watch the train moving around the track and to predict where the ball would land if it were dropped from the train when it reached the left edge of the cutout. The ball was not actually dropped.

The subjects made their predictions by placing a cup, $4 \mathrm{~cm}$ in diameter, on the floor where they thought it would catch the falling ball. Subjects' predictions were classified as straight down if the cup was placed less than $3.5 \mathrm{~cm}$ from the edge of the cutout. The subjects were then asked to explain their predictions. After each subject gave responses for all three conditions, the actual motions were shown, and the subject was questioned about any discrepancies between predictions and actual outcomes.

In all three conditions, the point at which the ball left the table was the same. In the Stationary condition, the ball had no forward motion and so, of course, fell straight down. In the Rolled and Carried conditions, the ball had a forward velocity of $45 \mathrm{~cm} / \mathrm{sec}$ when it left the table, so that in both conditions it traveled forward a distance of $20 \mathrm{~cm}$ before striking the ground.

The Stationary condition, which was included as a pretest to ensure that the subjects understood the task, was always administered first. Since all subjects responded correctly in this condition, it will not be considered further. The order of presentation of the Rolled and Carried conditions was counterbalanced across subjects.

\section{Results}

The results for the college-age subjects replicated previous findings (e.g., McCloskey et al., 1983). All of the college students knew that the ball rolled through the tube would continue moving forward as it fell. However, 7 of the students (35\%) thought that the ball dropped from the moving train would fall straight down. All of the adults who gave correct responses in the Carried condition had taken physics in high school or college.

The children's data present an interesting pattern, as shown in Figure 3. Almost all of the preschool and kindergarten children thought that the ball would fall straight down in both the Carried and the Rolled conditions. For 


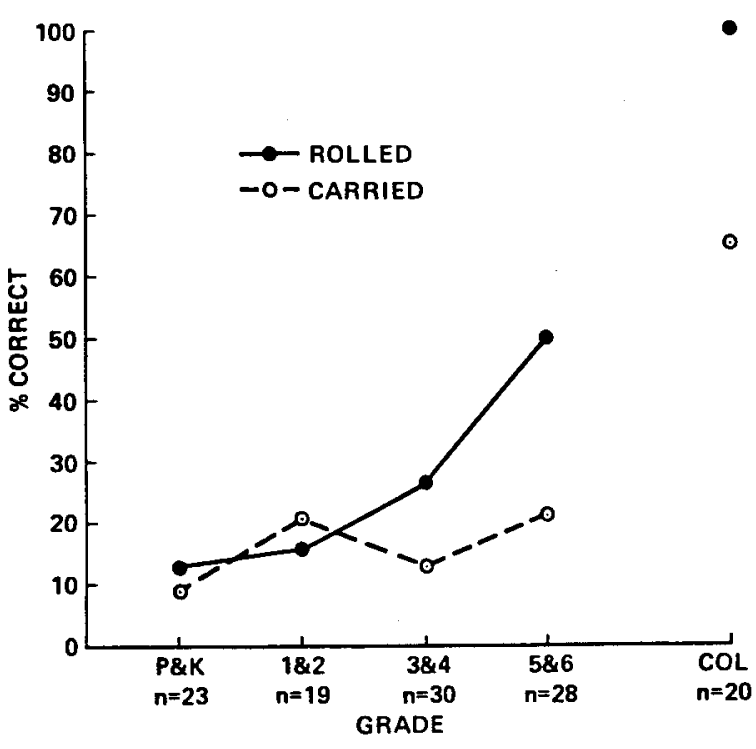

Figure 3. Proportion of subjects at each grade level who gave correct responses in the Rolled and the Carried conditions in Experiment 1.

the Rolled condition, with age, the children showed a steady increase in awareness that the ball would continue to move forward while falling. The percentage of subjects indicating that the ball rolled through the tube would continue forward increased from $9 \%$ for the preschoolers and kindergartners to $50 \%$ for the fifth and sixth graders. Grade level was significantly related to proportion of correct responses $\left[\chi^{2}(3)=10.59, p<.02\right]$. For the Carried condition, however, there was no significant improvement with age $\left[\chi^{2}(3)=2.13, p>.50\right]$. Only $21 \%$ of the fifth and sixth graders indicated that the ball dropped from the train would move forward while it fell. No effects of gender or order of condition presentation were found for either condition.

Subjects' predictions in the Rolled condition indicated that even those children and adults who realized the ball would continue forward as it fell tended to underestimate the distance the ball would travel before striking the ground. The mean estimate of $12.9 \mathrm{~cm}$ was significantly less than the actual distance of $20 \mathrm{~cm}[\mathrm{t}(48)=12.9$, $\mathrm{p}<.01]$. Among these subjects, there was no significant difference between the adults' and children's mean predictions [ 14.8 and $11.6 \mathrm{~cm}$, respectively; $\mathrm{t}(47)=1.27]$.

\section{EXPERIMENT 2}

We were concerned that the young children's failure to realize that the ball rolled through the tube would continue to move forward as it fell might reflect the specific conditions of our experiment (e.g., the relatively slow velocity of the ball, the use of a passive and hidden mechanism to start the ball moving), and not a general failure to realize that an object rolling from an elevated surface continues to move forward while falling. Hence, before attempting to interpret the findings of Experiment 1, we decided to test a limited number of children under conditions in which the rolled ball's initial forward motion was more salient in order to ensure that the children's straight down responses were not artifactual.

In this experiment we varied the speed of the rolling ball and the means by which it was set in motion. As in Experiment 1, children and adults predicted where the ball would land if it rolled through a tube and fell to the floor.

\section{Method}

Subjects. Eleven preschool through second-grade children (P-2; 6 boys and 5 girls), 11 third through sixth graders (3-6; 5 boys and 6 girls), and 17 college students (COL; 9 men and 8 women) participated in the study. The children were from middle-class families who responded to a solicitation in the university newspaper.

Apparatus and Procedure. Two launching mechanisms and three speeds of the ball were used. The passive-launch condition involved three clear plastic tubes with one end elevated to initiate the ball's motion. Three different elevations $(4.5,10.5$, and $19.0 \mathrm{~cm})$ were used to produce velocities approximately equal to, twice as great as, and three times as great as the velocity of the ball in Experiment 1 . Thus, for the three velocities, the ball traveled 23,43 , and $58 \mathrm{~cm}$ forward while falling. In the active-launch condition, a pinball-type spring-loaded piston was used to start the ball moving through the tube. Three different settings of the launcher were used to produce three velocities approximately equal to those in the passive-launch condition. In both conditions, the tubes were $85 \mathrm{~cm}$ in length and were mounted on a 1-m-high table with the tubes ending at the edge of the table.

The heights of the tubes and the tension of the spring launcher were always visible to the subject. For each launch type by speed combination, the subject viewed the launch of the ball and its movement to the edge of the table twice. On each occasion, the experimenter stopped the ball before it began to fall. After viewing the ball's motion, the subject predicted where the ball would have landed if it had been allowed to fall. The subjects indicated their predictions by marking the floor at the point where they thought the ball would hit.

Any prediction less than $3.5 \mathrm{~cm}$ from the point of launch (horizontally) was classified as straight down. The subjects were also asked to draw the trajectory of the ball on a $100 \times 70 \mathrm{~cm}$ drawing pad mounted perpendicular to the floor.

The subjects made predictions for all three velocities in each of the two launch mechanism conditions. The order of presentation of the two launching conditions was counterbalanced across subjects. Within each launching condition, the three speeds were presented in order from slow to fast.

\section{Results}

As in Experiment 1, a substantial proportion of the younger children thought that the ball would fall straight down after leaving the table. Table 1 presents for the three age groups the percentage of subjects predicting a straightdown path for the slow, medium, and fast speeds in the passive- and active-launch conditions. It can be seen from the table that, as in the first experiment, the percentage of straight-down predictions decreased as the age of the children increased $\left[\chi^{2}(1)=6.14, p<.05\right]$. The passiveversus active-launch manipulation had virtually no effect on the percentage of straight-down predictions $\left[\chi^{2}(1)=2.00, p>.10\right]$. The speed of the ball had a mar- 
Table 1

Percent of Subjects Predicting Straight-Down Trajectories for Passive- and Active-Launch Mechanisms in Experiment 2

\begin{tabular}{|c|c|c|c|c|c|c|}
\hline \multirow[b]{3}{*}{ Group } & \multicolumn{6}{|c|}{ Velocity } \\
\hline & \multicolumn{2}{|c|}{ Slow } & \multicolumn{2}{|c|}{ Medium } & \multicolumn{2}{|c|}{ Fast } \\
\hline & $\begin{array}{l}\text { Passive } \\
\text { Launch }\end{array}$ & $\begin{array}{l}\text { Active } \\
\text { Launch }\end{array}$ & $\begin{array}{l}\text { Passive } \\
\text { Launch }\end{array}$ & $\begin{array}{l}\text { Active } \\
\text { Launch }\end{array}$ & $\begin{array}{l}\text { Passive } \\
\text { Launch }\end{array}$ & $\begin{array}{l}\text { Active } \\
\text { Launch }\end{array}$ \\
\hline $\mathrm{P}-2$ & $88 \%$ & $55 \%$ & $66 \%$ & $55 \%$ & $66 \%$ & $44 \%$ \\
\hline $3-6$ & $66 \%$ & $44 \%$ & $33 \%$ & $33 \%$ & $22 \%$ & $22 \%$ \\
\hline $\mathrm{COL}$ & $0 \%$ & $0 \%$ & $0 \%$ & $0 \%$ & $0 \%$ & $0 \%$ \\
\hline
\end{tabular}

ginal effect in the passive-launch condition $\left[\chi^{2}(2)=5.21\right.$, $\mathrm{p}<.10]$, but no significant effect in the active-launch condition $\left[\chi^{2}(2)=2.36\right]$.

Among those children who did not give straight-down responses, all but 2 predicted either a parabolic path or an inverted-L path (i.e., the ball continues horizontally for some distance, then falls straight down). To be classified as parabolic, a subject's drawing needed to indicate that the ball began to fall as soon as it left the sup- port surface and fell at an increasingly greater speed. Predictions were classified as inverted- $L$ if the ball traveled some distance before beginning to fall and the transition from horizontal to vertical motion occurred abruptly. A comparison of these path forms indicates that, across ages, children who predicted parabolic paths were more accurate in judging the horizontal distance the ball travels before striking the ground. Figure 4 shows the actual distance, the average distance predicted by children employing inverted-L paths, and the average distance predicted by children's parabolic paths for the slow, medium, and fast velocities in the passive-launch condition. Across velocities, children using parabolic paths were closer to correct $[\mathrm{t}(10)=2.38, \mathrm{p}<.05]$. Furthermore, at all three velocities, children predicting inverted-L paths significantly underestimated the horizontal distance of the ball $[\mathrm{t}(4)=5.71, \mathrm{p}<.01$, for slow; $\mathrm{t}(4)=11.98$, $\mathrm{p}<.005$, for medium; and $\mathrm{t}(4)=17.68, \mathrm{p}<.005$, for fast]. Although children employing parabolic paths still tended to underestimate the horizontal distance, the un-

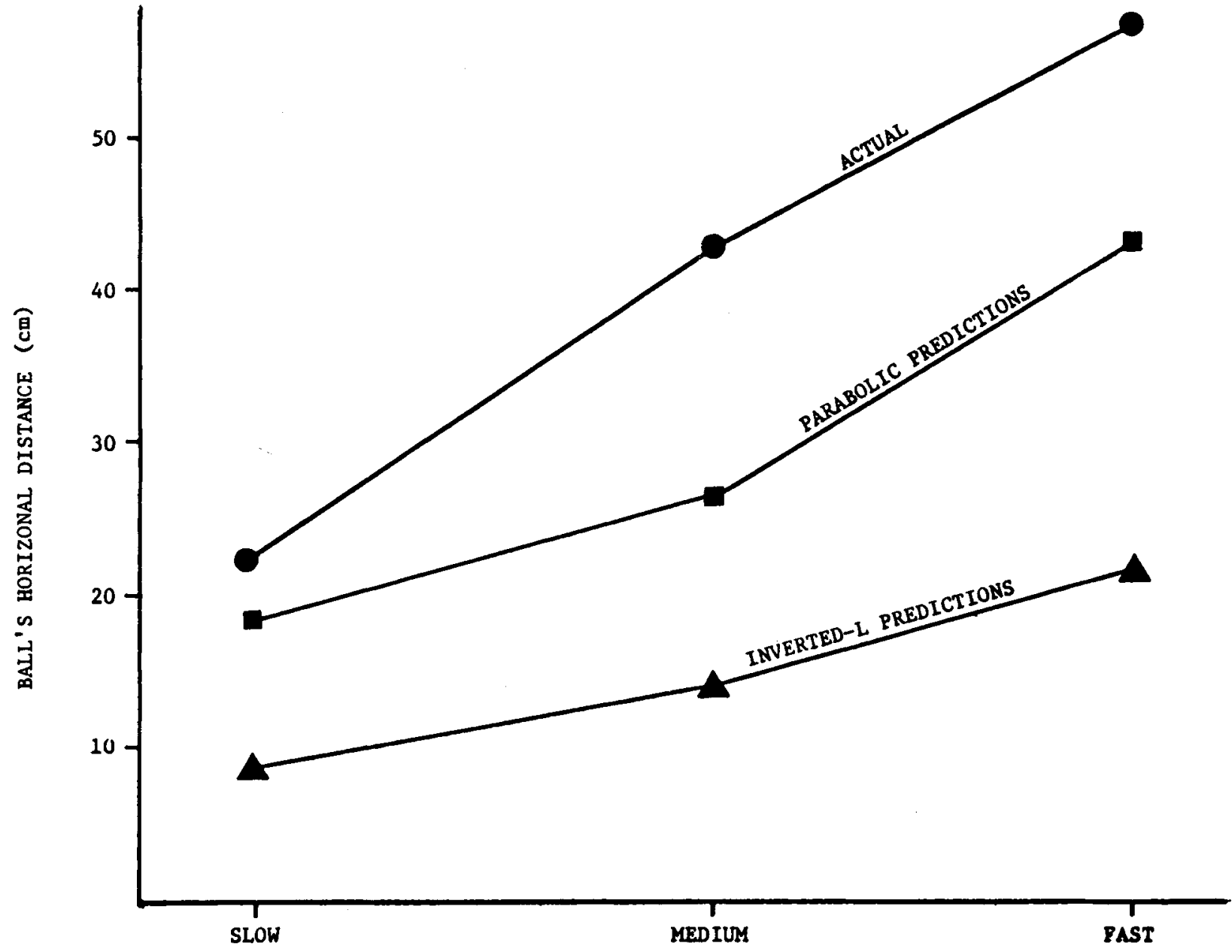

VELOCITY OF BALL IN PASSIVE LAUNCH CONDITION

Figure 4. Average horizontal distance predicted by children employing parabolic versus inverted-L paths compared with the actual distances in Experiment 2. 
Table 2

Number of Subjects Who Recognized the Monotonic Relation between Velocity and Horizontal Distance in Experiment 2

\begin{tabular}{|c|c|c|c|c|}
\hline \multirow[b]{3}{*}{ Group } & \multicolumn{4}{|c|}{ Launch Condition } \\
\hline & \multicolumn{2}{|c|}{ Passive } & \multicolumn{2}{|c|}{ Active } \\
\hline & Monotonic & Other & Monotonic & Other \\
\hline P-2 & 2 & $9(6)^{*}$ & 5 & $6(4)$ \\
\hline $3-6$ & 8 & $3(2)$ & 8 & $3(2)$ \\
\hline $\mathrm{COL}$ & 17 & 0 & 17 & 0 \\
\hline
\end{tabular}

*( ) indicates number who gave straight-down predictions for all three velocities.

derestimation is not significant $[\mathrm{t}(6)=.06$ for slow; $\mathrm{t}(6)$ $=2.09$ for medium; $t(6)=1.56$ for fast].

Lastly, we examined whether or not subjects recognized the monotonic relationship between the ball's velocity and the horizontal distance it travels before striking the ground. As shown in Table 2, older subjects were far more likely to recognize this relationship, in both the passive-launch $\left[\chi^{2}(2)=21.09, \mathrm{p}<.01\right]$ and active-launch $\left[\chi^{2}(2)=11.31, \mathrm{p}<.01\right]$ conditions.

\section{DISCUSSION}

The results of Experiment 2 demonstrate that the straight-down responses of the younger children in all conditions in Experiment 1 cannot be attributed to artifacts such as the low velocity of the ball or the passive nature of the launch mechanism. Many young children continued to demonstrate the belief that the ball would fall straight down when rolled off the edge of the table, despite our efforts to increase the salience of the ball's horizontal momentum. Furthermore, even those children who understood that such objects continue to move forward as they fall often held misconceptions concerning the trajectories, as well as the relationship between the horizontal velocity of the object and the distance it will travel before striking the ground.

Children's inability to think abstractly about the two determinants of the falling ball's motion (the ball's forward velocity, and gravity) may be linked to a general inability to perform mental combinations. Young children may focus their attention on the most salient vector operating on the object at any given time, much as they concentrate on the most salient dimension in physical conservation problems (Flavell, 1977). Thus, when the rolling ball reaches the edge of the table, the gravitational vector becomes very salient to the child, who realizes that in the absence of support objects invariably fall down. Even when children come to recognize that the gravitational vector does not immediately negate the ball's horizontal momentum, they tend to give responses that still fail to integrate the two vectors. Hence, they predict that the ball will continue horizontally for some distance, then fall straight down (i.e., the inverted-L path). Only those subjects who give responses that we have classified as parabolic understand that the ball will move both forward and downward at the same time, and integrate the two vectors successfully. Once such an integration is achieved, the consequence of increasing the magnitude of one vector (e.g., the ball's horizontal momentum) is much easier to comprehend. To develop an accurate understanding of the motion of an object in a particular type of situation, one must have not only the cognitive capabilities needed to understand the object's motion, but also appropriate perceptual experience with the motion. In the case of carried objects that are dropped, however, perceptual experience may be misleading rather than informative. The motion of an object's carrier becomes a moving reference frame when the object's fall is observed, thereby producing the perception of a "straight-down" fall.

Thus, our results show that children maintain the belief in a straight-down motion for a carried object even when they are cognitively capable of integrating an object's horizontal and vertical motions in the case of a rolling object. Understanding of the motion of objects dropped from a moving carrier, when it is achieved, appears to result primarily from formal instruction in physics.

One question that arises from the current findings is what young children perceive when viewing these events. We attempted to show the subjects in both experiments the actual outcomes of the events they predicted, and their reactions lend some insight. When the ball was released from the train, both the children and the adults who had given erroneous predictions were surprised that the ball did not fall straight down. Many concluded that the ball must have been released later than the experimenter had indicated; some suggested that the flatcar must have pushed the ball as it released it. A few did conclude that the ball kept moving forward as it fell, but their comments indicated that they could not explain the ball's motion.

Similarly, when shown the ball rolled off the table, some of the young children denied that it had moved forward as it fell and indicated a point beneath the table's edge as "where the ball really hit." Repeated demonstrations did convince some of these subjects that the ball continued to move forward as it fell. When these children were then asked to draw the trajectory of the ball, many gave inverted-L responses (the ball rolled out, then fell straight down), but a few did draw correct parabolic paths. Regardless of the path they drew, the children were unable to explain why the ball had fallen as it did, except to suggest that it was "going faster than I thought."

Clearly, it would be of interest to examine whether children who give straight-down predictions perceive straightdown trajectories as natural (and parabolic trajectories as anomalous) when viewing such events. Previous research suggests that people are often better at judging the natural outcome of events than at predicting what that outcome will be. For example, Shanon (1976) found that while many adults described free-falling objects as maintaining a constant velocity, most recognized an accelerating freefall as dynamically correct. Similarly, Kaiser and Proffitt (1984) demonstrated that children as young as 5 were sen- 
sitive to whether or not momentum is conserved in simple collision events only when they could observe the ongoing situation.

\section{CONCLUSION}

People's incorrect predictions concerning the outcome of simple mechanical events arise from several sources. First, as was found in research on the C-shaped-tube problem (Kaiser et al., in press), errors can result from the application of an erroneous belief about natural motions. In such situations, younger children who have not yet acquired this belief may actually perform better than older children. A second cause of error is to draw upon perceptual experiences which support erroneous beliefs. This is the case with the trajectory of objects dropped from moving bodies. Here, the moving reference frame illusion reinforces subjects' erroneous straight-down belief. Finally, when subjects are asked to solve motion problems in a representational context, cognitive limitations may constrain the subject's ability to integrate all relevant factors in the problem.

As previously demonstrated (Kaiser \& Proffitt, 1984; Shanon, 1976), a person's ability to judge the appropriateness of dynamics in ongoing visual displays may greatly outstrip his or her ability to reason abstractly about such situations. Although it may be inappropriate to suggest an autonomous relationship between "perceptual" and "conceptual" understandings, it does seem wise to recognize that a person may possess a perceptual appreciation of the natural dynamics of physical events, yet be unable to draw upon this knowledge when asked to conceptualize an event's outcome in a representational context.

\section{REFERENCES}

Champagne, A. B., Klopfer, L. E., A Anderson, J. H. (1980). Factors influencing the learning of classical mechanisms. American Journal of Physics, 48, 1074-1079.
Clement, J. (1982). Students' preconceptions in introductory mechanics. American Journal of Physics, 50, 66-71.

FlavelL, J. H. (1977). Cognitive development. Englewood Cliffs, NJ: Prentice-Hall.

Hochberg, J., \& Fallon, P. (1976). Perceptual analysis of moving patterns. Science, 194, 1081-1083.

Johansson, G. (1950). Configuration in event perception. Uppsala, Sweden: Almquist \& Wiksell.

Kaiser, M. K., McCloskey, M., \& Proffitt, D. R. (in press). Development of intuitive theories of motion: Curvilinear motion in the absence of external forces. Developmental Psychology.

Kaiser, M. K., \& ProffitT, D. R. (1984). The development of sensitivity to causally-relevant dynamic information. Child Development, 55, 1614-1624.

Kaiser, M. K., Proffitt, D. R., \& Anderson, K. (1985). Judgments of natural and anomalous trajectories in the presence and absence of motion. Journal of Experimental Psychology: Learning, Memory, \& Cognition, 11, 795-803.

MCCLOSKEY, M. (1983a). Intuitive physics. Scientific American, 248(4), 122-130.

MCCLOSKEy, M. (1983b). Naive theories of motion. In D. Gentner \& A. L. Stevens (Eds.), Mental models. Hillsdale, NJ: Erlbaum.

McCloskey, M., Caramazza, A., \& Green, B. (1980). Curvilinear motion in the absence of external forces: Naive beliefs about the motion of objects. Science, 210, 1139-1141.

MCCloskey, M., WashbuRn, A., \& FelCh, L. (1983). Intuitive physics: The straight-down belief and its origin. Journal of Experimental Psychology: Learning, Memory, \& Cognition, 9, 636-649.

Shanon, B. (1976). Aristotelianism, Newtonianism and the physics of the layman. Perception, 5, 241-243.

ShePard, R. N. (1981). Psychophysical complementarity. In M. Kubovy \& J. R. Pomerantz (Eds.), Perceptual organization. Hillsdale, NJ: Erlbaum.

SHEPARD, R. N. (1984). Ecological constraints on internal representations: Resonant kinematic of perceiving, imagining, thinking, and dreaming. Psychological Review, 91, 417-447.

VIENNOT, L. (1979). Spontaneous reasoning in elementary dynamics. European Journal of Science Education, 1, 205-221.

Wallach, H. (1959). The perception of motion. Scientific American, 201, 56-60.

Wallach, H. (1976). On perception. New York: Quadrangle/The New York Times Book Company.

(Manuscript received May 23, 1985; accepted for publication October 15,1985 .) 